\title{
Research Article \\ Equivalent Mechanical Model for Lateral Liquid Sloshing in Partially Filled Tank Vehicles
}

\author{
Zheng Xue-lian, Li Xian-sheng, and Ren Yuan-yuan \\ College of Traffic, Jilin University, No. 5988 Renmin Street, Changchun 130022, China \\ Correspondence should be addressed to Ren Yuan-yuan, maggie170101@gmail.com
}

Received 25 July 2012; Revised 17 September 2012; Accepted 10 October 2012

Academic Editor: Wuhong Wang

Copyright (C) 2012 Zheng Xue-lian et al. This is an open access article distributed under the Creative Commons Attribution License, which permits unrestricted use, distribution, and reproduction in any medium, provided the original work is properly cited.

This paper reports a new approach to investigating sloshing forces and moments caused by liquid sloshing within partially filled tank vehicles subjected to lateral excitations. An equivalent mechanical model is used in the paper to approximately simulate liquid sloshing. The mechanical model is derived by calculating the trajectory of the center of gravity of the liquid bulk in tanks as the vehicle's lateral acceleration changes from 0 to $1 \mathrm{~g}$. Parametric expressions for the model are obtained by matching the dynamic effect of the mechanical model to that of liquid sloshing. And parameter values of a liquid sloshing dynamic effect, such as sloshing frequency and forces, are acquired using FLUENT to simulate liquid sloshing in tanks with different cross-sections and liquid fill percentages. The equivalent mechanical model for liquid sloshing in tank vehicles is of a great significance for simplifying the research on roll stability of tank vehicles and for developing active/ passive roll control systems for these vehicles.

\section{Introduction}

Road tank vehicles are commonly used in carrying a wide range of liquid cargoes, mainly of a dangerous nature, such as chemical and petroleum products. At the same time, they are more frequently involved in rollover-related road accidents, which can seriously harm peoples and the environment. Statistical data collected by Statistique Canada have shown that $83 \%$ of lorry rollover accidents on highways are caused by tank vehicles [1]. And a US study has reported that the average annual number of cargo tank rollovers is about 1265 , which takes up $36.2 \%$ in the total number of heavy vehicle highway accidents [2].

Although there are many reasons that lead to tank vehicle rollover accidents, such as driver's fatigue, overtaking, bad road and weather conditions, and so forth, liquid sloshing in tanks is the main factor [3,4]. Due to different liquid densities and axle load limits on roads, tanks are in a partially filled state for the majority of the time. This phenomenon 
causes liquid sloshing in tanks when vehicle driving conditions change, meaning that strong sloshing forces are generated and vehicle roll stability is weakened. Therefore, research on liquid sloshing in partially filled tanks is one of the most important aspects when studying the roll stability of tank vehicles.

To date, many studies have been carried out on liquid flow and sloshing characteristics that happened in tanks, and the main methods can be summarized as follows.

(1) The quasi-static (QS) method. The cargo's static moment at a specified point on a tank vehicle can be approximated by calculating the transient center of gravity (CG) of the liquid bulk in the tank. Then, the liquid sloshing effect on tank walls can be analyzed. It is convenient and simple to obtain liquid sloshing force using the QS method. However, the analysis results have poor accuracy [5-7].

(2) The hydrodynamics method. By theoretically analyzing liquid flow characteristics in partially filled tanks, sloshing parameters can be acquired using basic hydrodynamic equations. Although the results so obtained are accurate, the analysis and the solution procedure are complicated. Due to the limited studies on turbulence and the fact that in reality the majority of flow can be categorized as turbulence, a large number of liquid flow phenomena cannot be explained using this method [8-12].

(3) The experimental method. By building a test platform or using test tank vehicles, liquid sloshing phenomenon can be observed and relevant parameters can be monitored by reproducing liquid sloshing $[13,14]$. The experimental results will depend on the test devices used, the sensor accuracy, and the operation of the tests, and so forth. And the method requires significant human and material resources.

(4) Computer simulation. Simulation software is used to simulate liquid sloshing and to obtain the values of a corresponding sloshing dynamic effect $[15,16]$.

(5) The equivalent mechanical model. Here, mechanical models are used to simulate liquid sloshing, which was created by NASA [17] and widely used for its simplicity and accuracy. Until now, most of the researches using this method have focused on spacecraft tanks and other vertical tanks [17-21]. Researches on horizontal tanks, such as those in tank vehicles, are limited [22-26].

By analyzing the present domestic and overseas conditions, the paper uses the equivalent mechanical model to simulate liquid sloshing in tank vehicles. The research outcomes have great importance for studying the roll stability of tank vehicles and for developing active/passive roll control systems for them.

\section{Derivation of the Equivalent Mechanical Model}

\subsection{Mathematical Form of the Mechanical Model}

Tanks with circular or oval cross-sections have larger volumes but the same surface area. Therefore, they are more popular in market applications and are the focus of study in this paper.

Theoretical analysis and experimental studies have shown that the first-order sloshing mode, which can be described by the oscillation of liquid-free surface, is the most important mode of liquid sloshing in partially filled tanks $[18,19]$. Therefore, we start the research by studying liquid sloshing in a partially filled tank with different liquid fill levels and solving for the trajectory of the CG of the liquid bulk. 
As shown in Figure 1, the tank cross-section is circular when $a / b=1$ and oval when $a / b>1$. In Figure 1, $a$ is a half of the tank width, $b$ is a half of the tank height, $h_{0}$ is the intersection point between the liquid level and the $y$-axis, and $\varphi$ is the tilt angle of the liquidfree surface.

Define the ratio of the height of the liquid level to the tank height as the liquid fill percentage or fill level in tanks, which can be expressed by

$$
\text { liquid fill percentage }=\frac{\left(h_{0}+b\right)}{2 b}=\Delta .
$$

Locus of the CG of the liquid bulk can be obtained from the following equations:

$$
\begin{gathered}
X=\frac{\int_{x_{1}}^{x_{2}} \int_{y_{1}}^{y_{2}} x d y d x}{\int_{x_{1}}^{x_{2}} \int_{y_{1}}^{y_{2}} d y d x} \\
Y=\frac{\int_{x_{1}}^{x_{2}} \int_{y_{1}}^{y_{2}} y d y d x}{\int_{x_{1}}^{x_{2}} \int_{y_{1}}^{y_{2}} d y d x} .
\end{gathered}
$$

And the cross-sectional area of the liquid in the tank can be expressed by

$$
A=\frac{3 a h_{0}}{b} \sqrt{b^{2}-h_{0}^{2}}+a b \arcsin \frac{\sqrt{b^{2}-h_{0}^{2}}}{b},
$$

when $h_{0}<0$, and

$$
A=\frac{a}{b}\left[h_{0} \sqrt{b^{2}-h_{0}^{2}}+b^{2}\left(\arcsin \frac{h_{0}}{b}+\frac{\pi}{2}\right)\right]
$$

when $h_{0}>0$.

The intersection point between the liquid-free surface and the $y$-axis, which is defined as $h$, changes with each tilt angle of the liquid-free surface. Therefore, the liquid-free surface in the $(x, y)$ coordinate system can be described by

$$
y=x \tan \varphi+h .
$$

The intersection points of the liquid-free surface with the tank periphery are given by

$$
\begin{aligned}
& \left(\frac{-a^{2} n h+a b \sqrt{a^{2} n^{2}+b^{2}-h^{2}}}{a^{2} n^{2}+b^{2}}, \frac{b^{2} h+a b n \sqrt{a^{2} n^{2}+b^{2}-h^{2}}}{a^{2} n^{2}+b^{2}}\right), \\
& \left(\frac{-a^{2} n h-a b \sqrt{a^{2} n^{2}+b^{2}-h^{2}}}{a^{2} n^{2}+b^{2}}, \frac{b^{2} h-a b n \sqrt{a^{2} n^{2}+b^{2}-h^{2}}}{a^{2} n^{2}+b^{2}}\right) .
\end{aligned}
$$




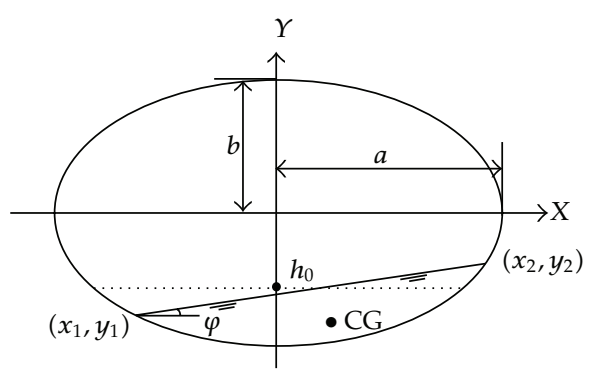

Figure 1: Schematic diagram for a partially filled tank with circular or oval cross-section.

The cross-sectional area of the liquid, which is defined as $S$, and its static moments on the $x$-axis and the $y$-axis, can be obtained from (2.3)-(2.6). And the acquired functions are all functions of $h$.

Regardless of the tilt angle of the liquid-free surface, the cross-sectional area of the liquid remains constant. Make $h$ vary within a given range with a quite small step size and calculate $S$ and its static moments at each value of $h$. Then, the CG of the liquid bulk can be obtained using (2.2), ensuring that the determinant condition of $|S-A| \leq \delta$ ( $\delta$ is a very small positive value depending on the step size of $h$ ) is satisfied.

The trajectory of the CG of the liquid bulk while the tilt angle of the liquid-free surface varies over a suitable range is shown in Figure 2, which shows that the trajectory of the CG of the liquid bulk remains parallel to the tank periphery.

In a vehicle's roll stability analysis using QS method, the liquid sloshing effect can be approximated by the static moment of the liquid bulk at a specified point on the tank vehicle [5-7]. The results have great errors from the actual condition, which cannot be neglected. However, simple mechanical devices, such as springs or pendulums, not only accurately calculate the liquid sloshing force and its influence on tank vehicles but also reflect liquid sloshing characteristics. For the problem discussed in this paper, the trammel pendulum, whose oscillation trajectory is an ellipse, is more appropriate; see [25].

\subsection{Equations of Motion for the Trammel Pendulum}

The oscillation trajectory and basic parameters of the trammel pendulum are shown in Figure 3. Suppose that the pendulum's oscillation trajectory is different from that of the CG of the liquid bulk, then $a_{p}+b_{p}$ is the arm length of the pendulum, where $a_{p}$ is a half of the major axis of the pendulum's oscillation trajectory and $b_{p}$ is a half of its minor axis. $a_{c g}$ is a half of the major axis of the elliptical trajectory of the CG of the liquid bulk and $b_{c g}$ is a half of its minor axis. $\theta$ is the pendulum amplitude, which is the maximum angle the pendulum swings away from the vertical position. And $\alpha$ is the angle between the line that connects the origin to the pendulum mass which is short for the mass of the bob on a pendulum and the $y$-axis.

The tank periphery, the oscillation trajectory of the pendulum, and the CG of the liquid bulk are all parallel to each other, which can be expressed as follows:

$$
\frac{a}{b}=\frac{a_{c g}}{b_{c g}}=\frac{a_{p}}{b_{p}}=\Lambda
$$




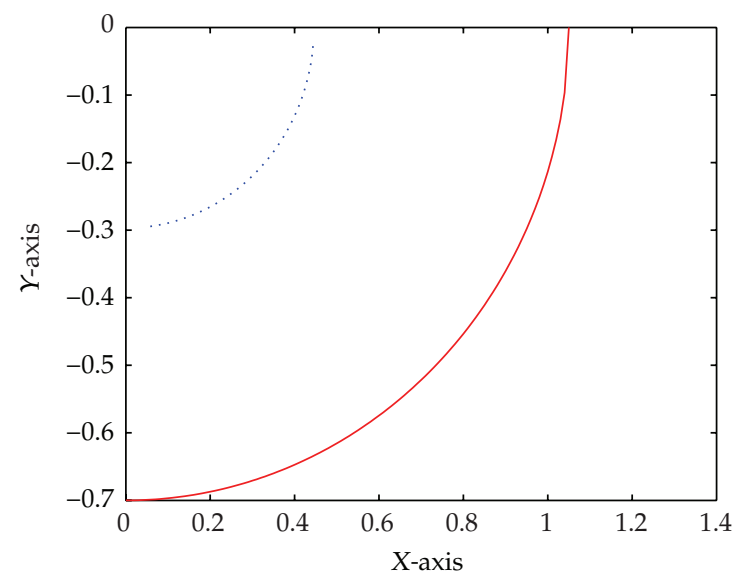

.... CG of the liquid bulk

- Tank periphery

Figure 2: The trajectory of the CG of the liquid bulk.

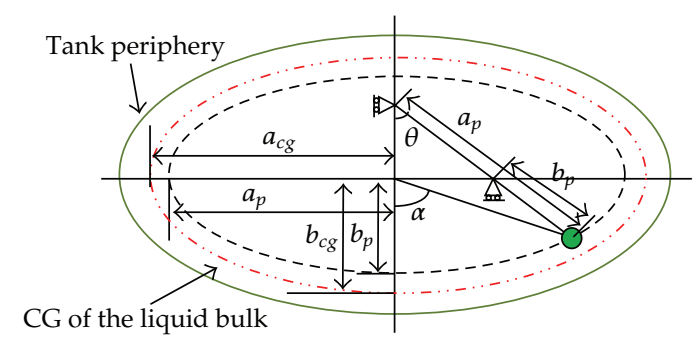

Figure 3: Schematic diagram for oscillation trajectory and basic parameters of the trammel pendulum.

The trammel pendulum's oscillation is affected by its arm length, amplitude, and the vehicle's lateral acceleration, not the pendulum mass.

The motion analysis for the trammel pendulum is shown in Figure 4, where $x y$ is the tank-fixed coordinate and $X Y$ is the earth-fixed coordinate. $l$ is the distance between the origin of $X Y$ and that of $x y$.

According to Figure 4, the absolute location of the pendulum mass can be expressed as

$$
\vec{r}=\left(a_{p} \sin \theta+l\right) \vec{i}-b_{p} \cos \theta \vec{j}
$$

Therefore, the velocity and acceleration of the pendulum mass can be expressed as follows:

$$
\begin{gathered}
\vec{r}=\left(a_{p} \dot{\theta} \cos \theta+i\right) \vec{i}+b_{p} \dot{\theta} \sin \theta \vec{j}, \\
\vec{r}=\left(\ddot{l}+a_{p} \ddot{\theta} \cos \theta-a_{p} \dot{\theta}^{2} \sin \theta\right) \vec{i}+b_{p}\left(\ddot{\theta} \sin \theta+\dot{\theta}^{2} \cos \theta\right) \vec{j} .
\end{gathered}
$$




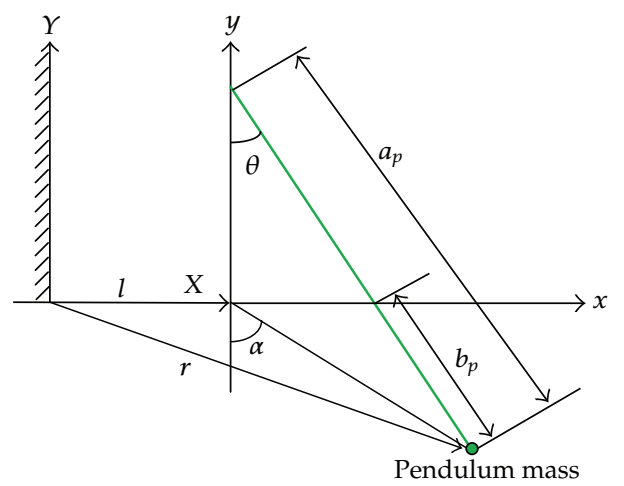

Figure 4: Diagram for motion analysis of the trammel pendulum.

The kinetic energy of the moving pendulum mass is defined by

$$
T=\frac{1}{2} m v^{2}=\frac{1}{2} m\left(a_{p}^{2} \dot{\theta}^{2} \cos ^{2} \theta+\dot{l}^{2}+2 a_{p} i \dot{\theta} \cos \theta+b_{p}^{2} \dot{\theta}^{2} \sin ^{2} \theta\right) .
$$

Assume that the zero of the potential energy is located at the surface of the equilibrium position of the trammel pendulum. Therefore, the gravitational potential energy of the trammel pendulum can be expressed as

$$
Q=m g b_{p}(1-\cos \theta)
$$

According to (2.11)-(2.12), a Lagrangian function can be used to obtain the kinetic equation for the pendulum system, which can be written as follows:

$$
L=T-Q=\frac{1}{2} m\left(a_{p}^{2} \dot{\theta}^{2} \cos ^{2} \theta+\dot{l}^{2}+2 a_{p} i \dot{\theta} \cos \theta+b_{p}^{2} \dot{\theta}^{2} \sin ^{2} \theta\right)+m g b_{p}(\cos \theta-1)
$$

The motion of the trammel pendulum system can be expressed by

$$
\frac{\partial}{\partial t}\left(\frac{\partial L}{\partial \dot{\theta}}\right)-\frac{\partial L}{\partial \theta}=0
$$

where

$$
\begin{gathered}
\frac{\partial L}{\partial \dot{\theta}}=m\left(a_{p}^{2} \dot{\theta} \cos ^{2} \theta+a_{p} \dot{l} \cos \theta+b_{p}^{2} \dot{\theta} \sin ^{2} \theta\right) \\
\frac{\partial}{\partial t}\left(\frac{\partial L}{\partial \dot{\theta}}\right)=m\left(a_{p}^{2} \ddot{\theta} \cos ^{2} \theta-a_{p}^{2} \dot{\theta}^{2} \sin 2 \theta+a_{p} \ddot{l} \cos \theta-a_{p} \dot{i} \dot{\theta} \sin \theta+b_{p}^{2} \ddot{\theta} \sin ^{2} \theta+b_{p}^{2} \dot{\theta}^{2} \sin 2 \theta\right) \\
\frac{\partial L}{\partial \theta}=m\left(-0.5 a_{p}^{2} \dot{\theta}^{2} \sin 2 \theta-a_{p} \dot{i} \dot{\theta} \sin \theta+0.5 b_{p}^{2} \dot{\theta}^{2} \sin 2 \theta\right)-m g b_{p} \sin \theta
\end{gathered}
$$




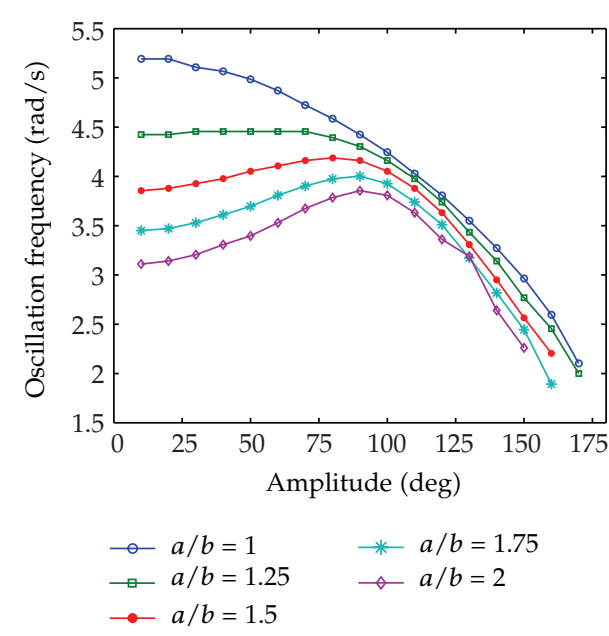

(a) Oscillation frequencies

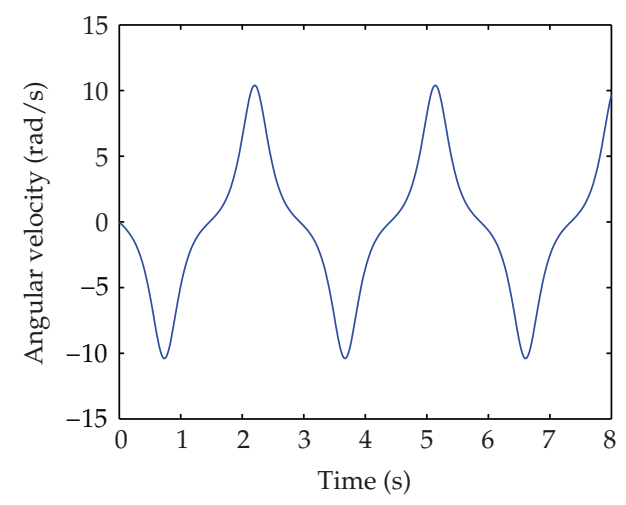

(c) Angular velocity when amplitude is 170 degrees

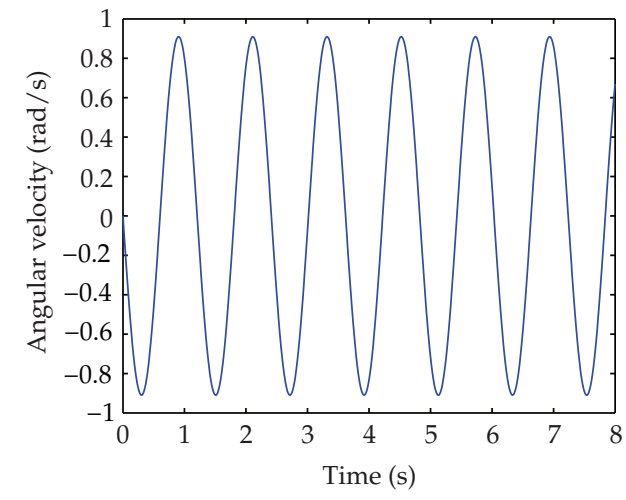

(b) Angular velocity when amplitude is 10 degrees

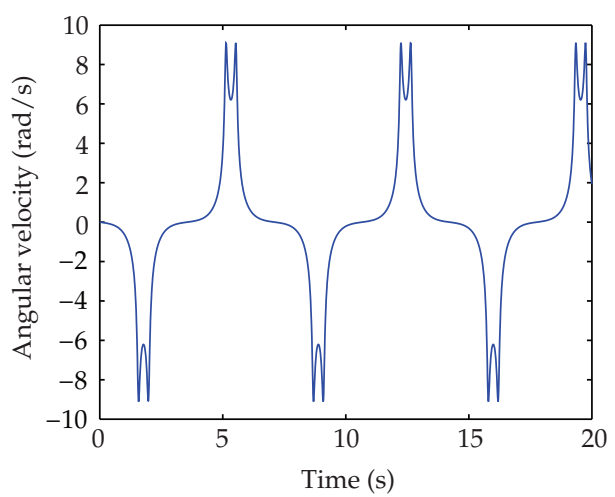

(d) Angular velocity when amplitude is 179 degrees

Figure 5: Motion characteristics of trammel pendulum.

Substituting (2.16) into (2.14) we get

$$
\left(a_{p}^{2} \cos ^{2} \theta+b_{p}^{2} \sin ^{2} \theta\right) \ddot{\theta}+\frac{1}{2}\left(b_{p}^{2}-a_{p}^{2}\right) \dot{\theta}^{2} \sin 2 \theta+g b_{p} \sin \theta+\ddot{l} a_{p} \cos \theta=0 .
$$

MATLAB's ODE algorithm is used to solve (2.17). During the solution procedure, we make $a / b$ varies between 1 and 2 with a 0.25 step size and the pendulum amplitude varies between 10 degrees and 180 degrees with a 10-degrees step size.

Oscillation frequencies and angular velocities for pendulums with small and large amplitudes are presented in Figure 5. Tanks with different cross-sections have the same crosssectional area and $a=b=0.3602 \mathrm{~m}$ when the cross-section is circular $(a / b=1)$.

As Figure 5(a) shows the pendulum's oscillation frequency depends on its arm length and amplitude, the oscillation frequency decreases with an increase in amplitude when $a / b=1$. However, for the other pendulums, the oscillation frequency rises with an increase in amplitude, reaching the maximum frequency when the amplitude reaches a certain value, and then decreasing after that. For instance, the maximum frequency appears at an amplitude 


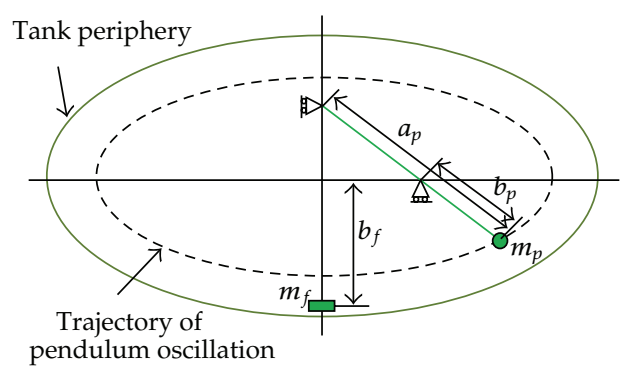

Figure 6: Parameters to be determined for the pendulum.

of 90 degrees when $a / b=2$. For all pendulums, the oscillation frequency remains almost constant when the amplitude is below a certain value.

As seen in Figures 5(b)-5(d), with an increase in amplitude, the motion of the pendulum becomes irregular with more nonlinearity, especially for the amplitude larger than 170 degrees. Fortunately, the lateral acceleration of the tank vehicles is smaller than $0.45 \mathrm{~g}$ in reality to avoid vehicle rollovers, which means that the tilt angle of the liquid-free surface is always smaller than 90 degrees. Therefore, the nonlinear characteristics of the pendulum can be neglected and the pendulum can be assumed approximately linear.

\section{Parametric Expressions for the Trammel Pendulum Model}

The parameters that need to be determined for the pendulum are presented in Figure 6, where $m_{p}$ is the pendulum mass, $m_{f}$ is the fixed liquid mass, and $b_{f}$ is the distance between the center of the ellipse and the location of the fixed liquid mass.

Due to the fact that not all of the liquid participate in the sloshing [11-14, 17, 18, 20], the pendulum parameters $a_{p}, b_{p}$, and $m_{p}$ are not equal to $a_{c g}, b_{c g}$, and the liquid mass $m$, respectively.

Because the pendulum parameters cannot be obtained directly, and taking into consideration that the pendulum parameters must have relations with the liquid sloshing parameters, analogy method is used in obtaining parametric expressions for the mechanical model.

\subsection{Derivation of the Pendulum Arm Length Parameters}

According to Section 2.2, the pendulum's oscillation frequency partly depends on its arm length which can be expressed as $a_{p}+b_{p}$. For a partially filled tank with a specified crosssection, the liquid sloshing frequency is known and, using (2.7), $a_{p}$ and $b_{p}$ can be obtained. Therefore, (2.7) and (2.17) are sufficient to derive the pendulum arm length parameters.

Given the fact that the oscillation frequency remains almost constant when the pendulum amplitude is quite small, define the following quantities:

$$
\begin{gathered}
\sin \theta \approx \theta, \quad \cos \theta \approx 1, \quad \sin ^{2} \theta \approx 0, \\
\cos ^{2} \theta \approx 1, \quad \sin 2 \theta \approx 2 \theta .
\end{gathered}
$$


Then, (2.17) can be rewritten as follows (see in [25]):

$$
\ddot{\theta}+\left(\frac{b_{p}^{2}-a_{p}^{2}}{a_{p}^{2}}\right) \dot{\theta}^{2} \theta+\frac{g b_{p}}{a_{p}^{2}} \theta=0
$$

For analytical simplicity, define the following quantities:

$$
\theta=x ; \quad \frac{d x}{d t}=y
$$

Then, (3.2) can be rewritten as follows:

$$
\frac{d y}{d t}=C_{1} y^{2} x-C_{2} x
$$

where $C_{1}=-\left(\left(b_{p}^{2}-a_{p}^{2}\right) / a_{p}^{2}\right), C_{2}=g b_{p} / a_{p}^{2}$.

Now, the orientation field equation for the trammel pendulum can be expressed as

$$
\frac{d y}{d x}=\frac{C_{1} y^{2} x-C_{2} x}{y}
$$

Equation (3.5) can be transformed into the following form:

$$
\frac{y d y}{C_{1} y^{2}-C_{2}}=x d x
$$
as follows:

By solving (3.6), the phase plane trajectory equation for the pendulum can be written

$$
y=\frac{ \pm \sqrt{C_{1}\left(C_{2}+A e^{C_{1} x^{2}}\right)}}{C_{1}}
$$

where $A$ is an integral constant that can be obtained by setting $x=x_{\max }$ and $y=0$.

Substituting $A$ into (3.7) gives

$$
y=\frac{ \pm \sqrt{C_{1} C_{2}\left(1-e^{C_{1}\left(x^{2}-x_{\max }^{2}\right)}\right)}}{C_{1}} .
$$

Based on (3.8), the phase trajectories for trammel pendulums with different amplitudes are presented in Figure 7. It is concluded that the pendulum system moves back and forth and keeps a circular motion with the same amplitude. 
Now, define the following quantities for (3.8):

$$
C_{3}=\sqrt{\frac{C_{2}}{C_{1}}} ; \quad z=C_{1}\left(x^{2}-x_{\max }^{2}\right) .
$$

Then, (3.8) can be rewritten as follows:

$$
y=C_{3}\left(1-e^{z}\right)^{1 / 2}
$$

Rewriting (3.10) using a Taylor series expansion and neglecting higher order terms gives

$$
y=C_{3} \sqrt{C_{1}\left(x_{\max }^{2}-x^{2}\right)}=\sqrt{\frac{g b_{p}}{a_{p}^{2}}} \sqrt{x_{\max }^{2}-x^{2}} .
$$

At the instance of tanks with circular cross-section, the coefficient of (3.11) is equal to $\sqrt{g / a_{p}}$, which is the frequency expression for simple pendulums. Therefore, the phase plane trajectory equation of a simple pendulum is thus given by

$$
y=\omega \sqrt{x_{\max }^{2}-x^{2}}
$$

Comparing (3.11) to (3.12), the natural oscillation frequency of the trammel pendulum with small amplitude can be expressed as follows:

$$
\omega=\sqrt{g b_{p} / a_{p}^{2}}
$$

The oscillation frequencies of pendulums with small amplitudes obtained from (2.17) are used to verify the accuracy of (3.13). The results show that (3.13) is very consistent with (2.17).

As the liquid sloshing frequency in a partially filled tank vehicle is already known, $a_{p}$ and $b_{p}$ can easily be obtained based on (2.7) and (3.13).

\subsection{Derivation of the Pendulum Mass Parameters}

The lateral liquid sloshing force in a tank vehicle is caused by the liquid mass that participates in the sloshing. When a pendulum is used to simulate the liquid sloshing, the liquid mass that participates in the sloshing is equal to the pendulum mass. According to the law of conservation of mass, the liquid mass that does not participate in the sloshing is equal to the fixed part.

A direct solution for the pendulum mass is difficult and requires hydrodynamic theory analysis. Thus, an alternative method is used.

First of all, suppose that all of the liquid mass participate in the sloshing. If the maximum lateral acceleration of the liquid bulk is known, then the sloshing force of the entire 


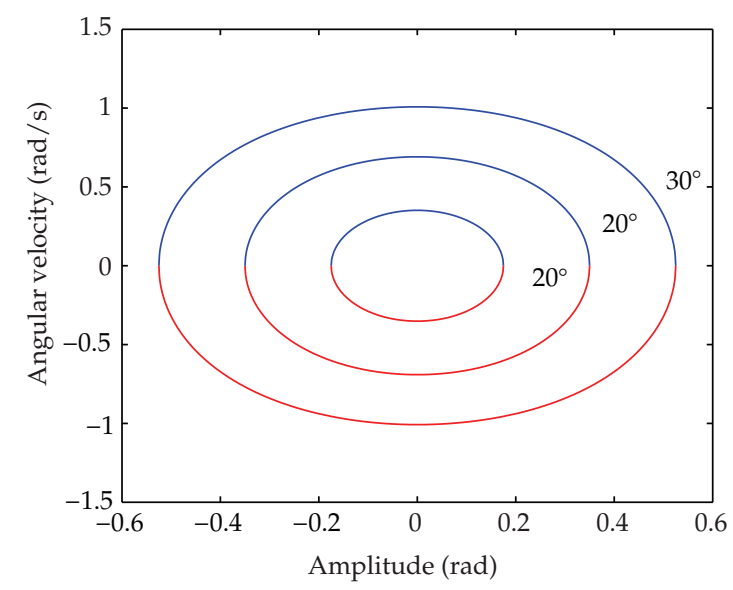

Figure 7: Phase trajectories for trammel pendulum with different amplitudes.

liquid mass can be obtained. By comparing it with the actual sloshing force, the ratio of the pendulum mass to the entire liquid mass can be acquired. Since the entire liquid mass is known, the pendulum mass can thus be calculated.

According to Newton's second law, the sloshing force can be expressed by

$$
F_{t}=\max \left[m a_{y}\right]
$$

where $F_{t}$ is the sloshing force caused by the entire liquid mass, $a_{y}$ is the maximum lateral acceleration of the liquid bulk, and $m$ is the entire liquid mass.

Assume that the tank length is $1 \mathrm{~m}$ and the liquid density is known. Then, given the size of the tank cross-section and the value of the liquid fill percentage, $m$ can be obtained.

Make the lateral acceleration of the tank vehicle equal to zero and liquid oscillate only under the action of gravity. According to (2.10), the maximum lateral acceleration of the liquid can be expressed as

$$
a_{y}=\max \left[a_{p}(-\dot{\theta} \sin \theta+\ddot{\theta} \cos \theta)\right] .
$$

From (3.14)-(3.15), the maximum sloshing force caused by the entire liquid mass can be obtained.

The actual sloshing force is given by

$$
F_{p}=\max \left[m_{p} a_{y}\right]
$$

Equation (3.16) divided by (3.14) gives

$$
\frac{m_{p}}{m}=\frac{F_{p}}{F_{t}} .
$$


Then, the fixed liquid mass is

$$
m_{f}=m-m_{p}
$$

No matter where the locations of $m_{f}$ and $m_{s}$ are, the action point of the two parts always coincides with that of the CG of the entire liquid mass. Therefore, the sum of the static moments of $m_{f}$ and $m_{s}$ at the lowest point of the tank is equal to that of $m$ at the same point when the liquid-free surface is level, which can be expressed by

$$
m_{f}\left(b-b_{f}\right)+m_{p}\left(b-b_{p}\right)=m\left(b-b_{c g}\right) .
$$

If all the other parameters are already known, then $b_{f}$ can be obtained from (3.19).

\section{Simulation and Discussion}

\subsection{Settings for FLUENT Simulation Conditions}

Based on Section 3.1, parameters used to describe the liquid sloshing dynamic effect, such as the sloshing frequency and the maximum lateral sloshing force, will be obtained in this section to completely specify the equivalent mechanical model.

The FLUENT software is used to simulate liquid sloshing that occurs in tank vehicles and to obtain the values of relevant parameters. Before performing the simulation, the sizes of tank cross-sections should be decided and the corresponding simulation conditions should be set.

According to a market survey, the cross-sectional area of oval tanks is usually just under $2.4 \mathrm{~m}^{2}$. XH9140G, a typical tank semitrailer of PieXin brand, is chosen as the simulation object [27]. The long axis of the tank cross-section is $2.3 \mathrm{~m}$ and the short one is $1.3 \mathrm{~m}$; the tank's wall thickness is neglected. According to the principle that tanks with different cross-sections have the same surface area, the sizes of the tank cross-sections are presented in Table 1.

The tilt angle of the liquid-free surface is set to be 5 degrees to maintain the linear characteristics of the pendulum and to ensure that the liquid will oscillate gently under the action of gravity. Water is chosen as the simulation liquid. The liquid fill percentage is set to vary from $10 \%$ to $90 \%$ with a $10 \%$ step size.

The maximum velocity of water can be obtained when it moves to the lowest position in the tank, which is presented as follows:

$$
v=\sqrt{2 g \Delta h}
$$

where $v$ is the water's velocity, and $\Delta h$ is the vertical distance that the CG of the water bulk moves.

Suppose that $b_{c g}=0.2$, which is a quite small value compared with $b$, the flow Reynolds number can be expressed as follows:

$$
\operatorname{Re}=\frac{D v}{v}=2.44 \times 10^{5} \gg 2000
$$


Table 1: Sizes of tank cross-sections (unit: $\mathrm{m}$ ).

\begin{tabular}{cccccc}
\hline & $a / b=1$ & $a / b=1.25$ & $a / b=1.5$ & $a / b=1.75$ & $a / b=2$ \\
\hline$a$ & 0.857 & 0.9585 & 1.05 & 1.134 & 1.2124 \\
$b$ & 0.857 & 0.7668 & 0.7 & 0.6481 & 0.6062 \\
\hline
\end{tabular}

According to (4.2), the liquid sloshing that occurs in the tank vehicles can be categorized as turbulence.

Although the liquid velocity in the region near to the wall is quite low and its order of magnitude is around $10^{-2}$, the turbulence characteristic of the water flow is still quite apparent. In order to choose the standard wall function for the near-wall treatment of the viscous model, the meshing for the tank model must be qualified and the wall $Y$-plus, which is the dimensionless distance between the CG of the first layer of the grid and the wall, should be within the range from 10 to 100 .

Based on real-life conditions, the reference pressure location for the operating conditions is in the pressure inlet and the gravity is $9.81 \mathrm{~m} / \mathrm{s}^{2}$ in the negative direction of the $y$-axis.

The intensity and hydraulic diameter are chosen as the turbulence specification method. For the liquid that oscillates freely under the action of gravity generated by the small tilt angle of the liquid-free surface, the turbulence intensity will be within the range $0.1 \%-0.5 \%$.

The hydraulic diameter is calculated as follows:

$$
D=\frac{4 A}{x}
$$

where $D$ is the hydraulic diameter, $A$ is the cross-sectional area of the liquid, and $X$ is the wetted perimeter.

The PISO algorithm and the Body Force Weighted method are chosen for the pressurevelocity coupling and the pressure discretization, respectively; see [28].

According to the above settings, a schematic diagram for the liquid sloshing model can be obtained and is shown in Figure 8 .

To obtain the cycle time of the liquid sloshing, a point located in the tank that will always be immersed in the liquid is specified. Then, the cycle time of the liquid sloshing can be obtained by monitoring the lateral velocity of this point.

\subsection{Simulation Results}

The cycle time of the lateral velocity of a point $(-0.84,0)$ in a tank with circular cross-section and $20 \%$ liquid fill level is presented in Figure 9.

The relation between the cycle time and the angular frequency is given by

$$
\omega=\frac{2 \pi}{T}
$$




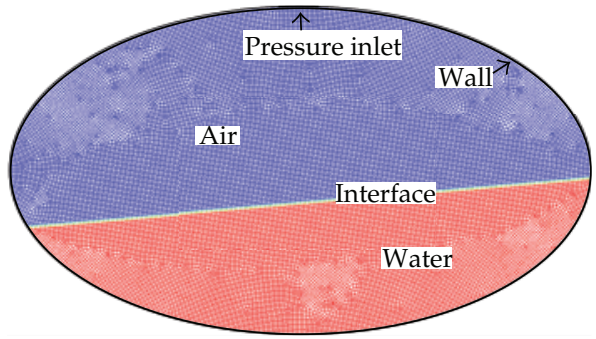

Figure 8: Schematic diagram for fluid sloshing model.

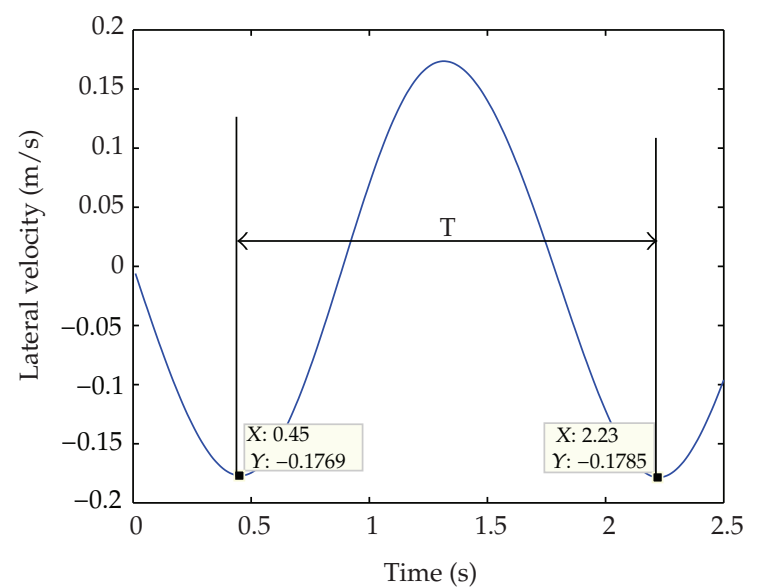

Figure 9: Lateral velocity of a point in a tank with circular cross-section and $20 \%$ liquid fill level.

The natural frequencies of liquid sloshing in tanks with different cross-sections and liquid fill levels are obtained using (4.4) and plotted in Figure 10.

Equation (3.13) can be rewritten as follows:

$$
\frac{b_{p}}{b}=\frac{g}{\omega^{2} r^{2} b}
$$

As the sloshing frequencies are already known, $b_{p} / b$ is obtained and plotted in Figure 11.

Curves fitting is done to the data points in Figure 11 to obtain an equation that describes $b_{p} / b$ as a function of the fill percentage and the tank cross-section. This equation is as follows:

$$
\begin{aligned}
\frac{b_{p}}{b}= & 1.089+0.726 \Delta-0.1379 \Lambda-0.953 \Delta^{2}-1.216 \Lambda \Delta \\
& +0.05141 \Lambda^{2}-0.06107 \Delta^{3}+0.5739 \Lambda \Delta^{2}+0.1632 \Lambda^{2} \Delta .
\end{aligned}
$$

The curves specified by (4.6) are plotted in Figure 12. And the relative error of the curves fitting for $b_{p} / b$ is plotted in Figure 13. 


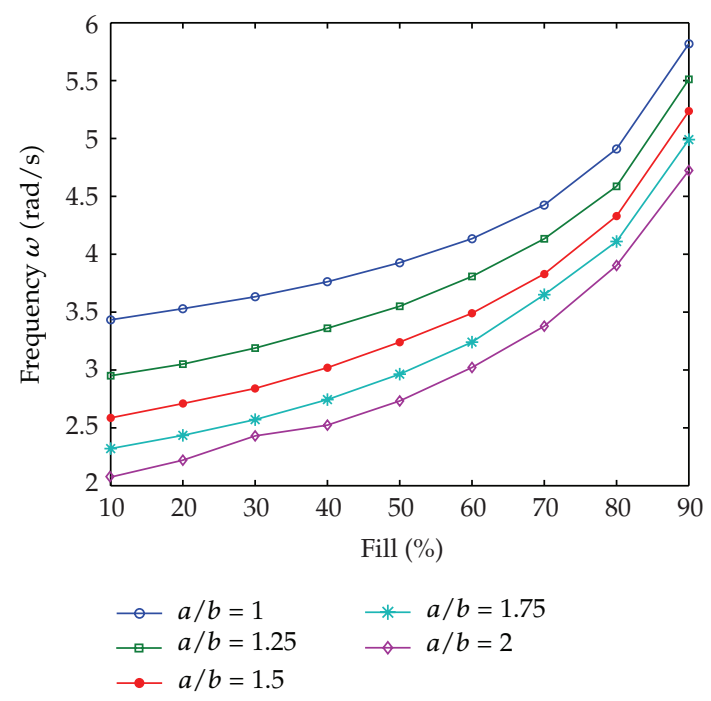

Figure 10: Natural frequencies of liquid sloshing.

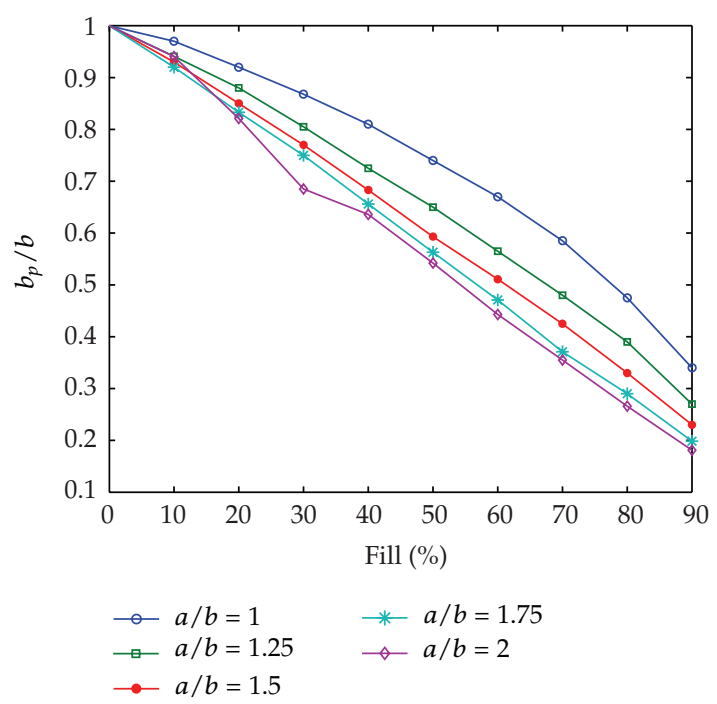

Figure 11: Values of $b_{p} / b$.

$a_{p}$ can be obtained from (2.7) and (4.6). As a result, all of the pendulum arm length parameters have been obtained.

The maximum sloshing force during the liquid sloshing process can be obtained by monitoring the lateral force coefficient for the tank walls, and the results are presented in Figure 14. This shows that the maximum sloshing force is generated when the liquid fill percentage is close to $60 \%$. Therefore, for tank vehicles, a liquid fill percentage close to $60 \%$ is the worst laden state. 


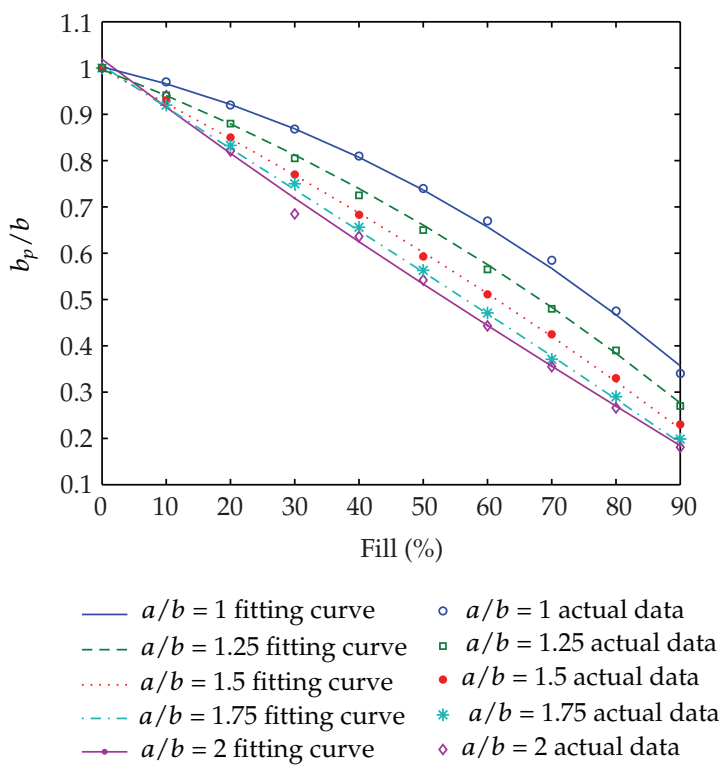

Figure 12: Fitting curves for $b_{p} / b$.

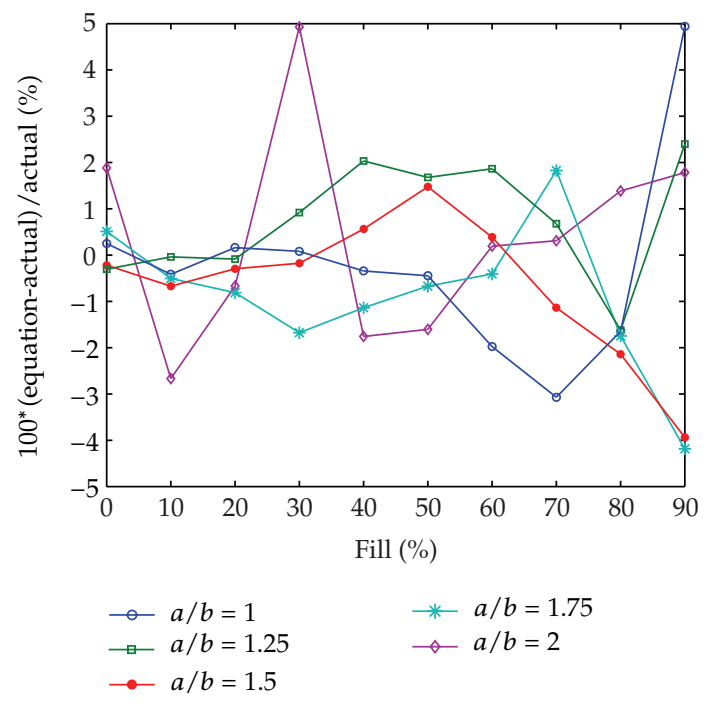

Figure 13: The relative error of the curves fitting for $b_{p} / b$.

The ratio of the sloshing force to the liquid mass is presented in Figure 15. This shows that the lower is the liquid fill percentage; the larger is the sloshing force generated by per unit of liquid mass.

To solve the sloshing force of the entire liquid mass, the maximum lateral acceleration is needed. According to (2.17), the pendulum amplitude is needed to be obtained at first. 


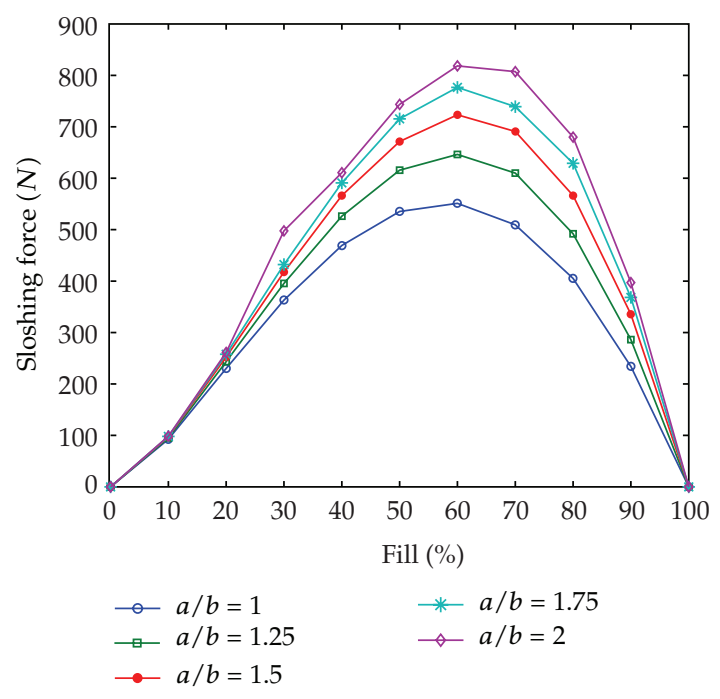

Figure 14: Maximum sloshing forces.

Table 2: Pendulum amplitudes in tanks with different cross-sections.

\begin{tabular}{cccccc}
\hline & $a / b=1$ & $a / b=1.25$ & $a / b=1.5$ & $a / b=1.75$ & $a / b=2$ \\
\hline$\alpha$ & 5 & 7.788 & 11.133 & 15 & 19.287 \\
$\theta$ & 5 & 6.244 & 7.474 & 8.705 & 9.925 \\
\hline
\end{tabular}

However, the pendulum arm is not perpendicular to the liquid-free surface, except for tanks with a circular cross-section. According to Figure 4, the following equation can be obtained:

$$
\theta=\tan ^{-1}\left(\frac{b}{a} \tan \alpha\right)
$$

$\alpha$ that exist in (4.7) can be obtained by solving the CG of the liquid bulk. For 5 degrees tilt angle of the liquid-free surface, $\alpha$ and $\theta$ are as listed in Table 2.

The maximum lateral acceleration can be obtained using (2.17), (3.15), and Table 2. Then, the lateral sloshing force for the entire liquid mass can be calculated using (3.14). Finally, $m_{p} / m$ can be derived from (3.17) and these values are presented in Figure 16.

Curves fitting is done to the points in Figure 16 to obtain an equation that describes $m_{p} / m$ as a function of the tank cross-section and the liquid fill percentage. The fitted equation is given by the following expression:

$$
\begin{aligned}
\frac{m_{p}}{m}= & 0.7844-1.729 \Delta+0.3351 \Lambda+1.156 \Delta^{2}+0.7256 \Lambda \Delta \\
& -0.1254 \Lambda^{2}-0.3219 \Delta^{3}-0.9152 \Lambda \Delta^{2}+0.08043 \Lambda^{2} \Delta .
\end{aligned}
$$

The curves given by (4.8) are plotted in Figure 17. And the relative error of the curves fitting for $m_{p} / m$ is plotted in Figure 18 .

Given the pendulum mass, the fixed liquid mass can be obtained using (3.18). 


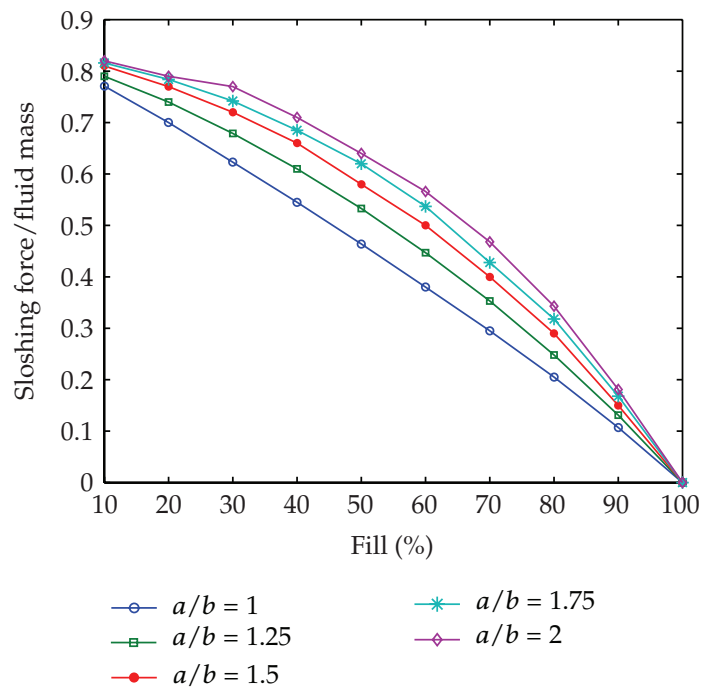

Figure 15: Sloshing force generated by per unit of liquid mass.

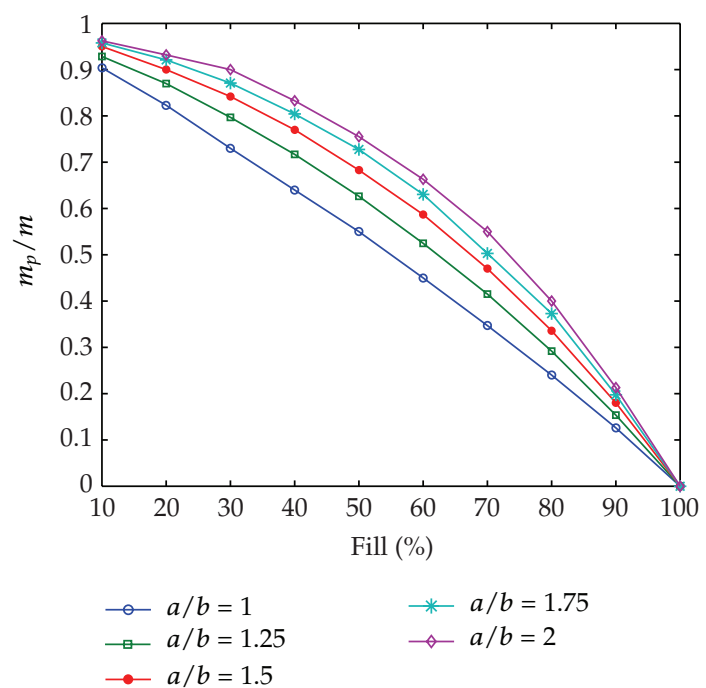

Figure 16: Values of $m_{p} / m$.

According to (3.19), the position of the fixed liquid mass is given by

$$
\frac{b-b_{f}}{b}=\frac{m\left(b-b_{c g}\right)-m_{f}\left(b-b_{p}\right)}{m_{f} b} .
$$

The curves given by (4.9) are presented in Figure 19. It shows that the position of the fixed liquid mass is close to the center of the ellipse, except when the liquid fill percentage is below $30 \%$. And some points that are derived apparently from the equation curve are marked 


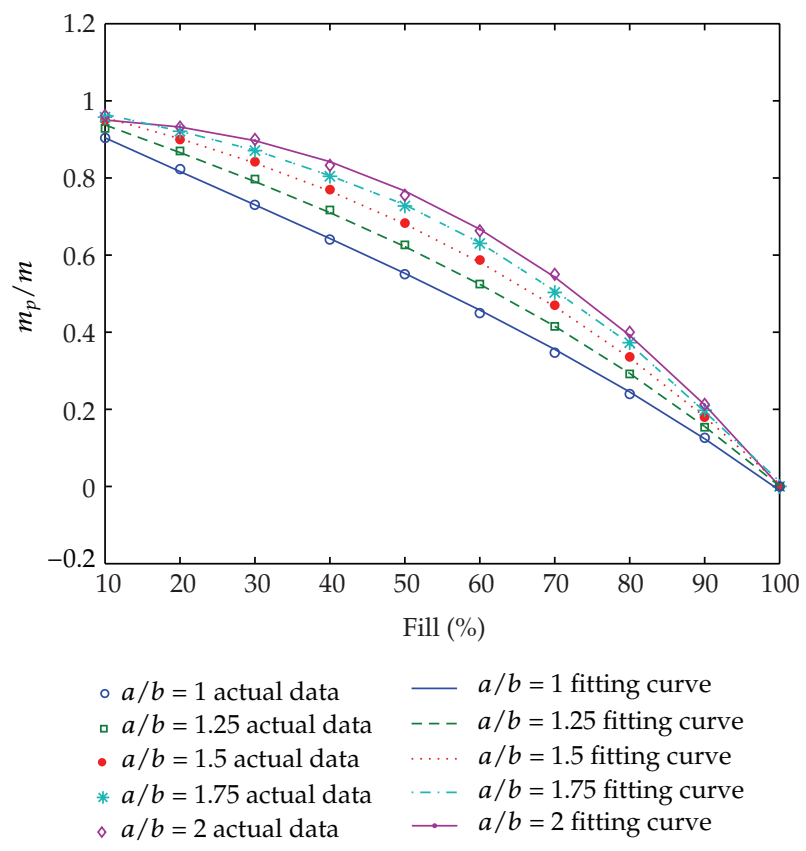

Figure 17: Fitting curves for $m_{p} / m$.

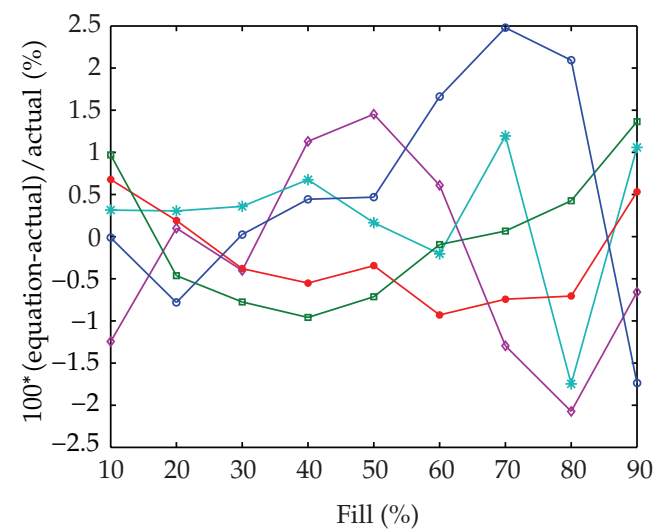

$$
\begin{aligned}
\rightarrow a / b & =1 & \rightarrow & a / b=1.75 \\
\square & a / b=1.25 & \rightarrow & a / b=2 \\
\rightarrow a / b & =1.5 & &
\end{aligned}
$$

Figure 18: The relative error of the curves fitting for $m_{p} / m$.

in Figure 19. The reason why this phenomenon happens should be investigated in a future study.

\subsection{Conclusions}

To deal with the complexity of analyzing a liquid sloshing dynamic effect in partially filled tank vehicles, the paper uses equivalent mechanical model to simulate liquid sloshing. 


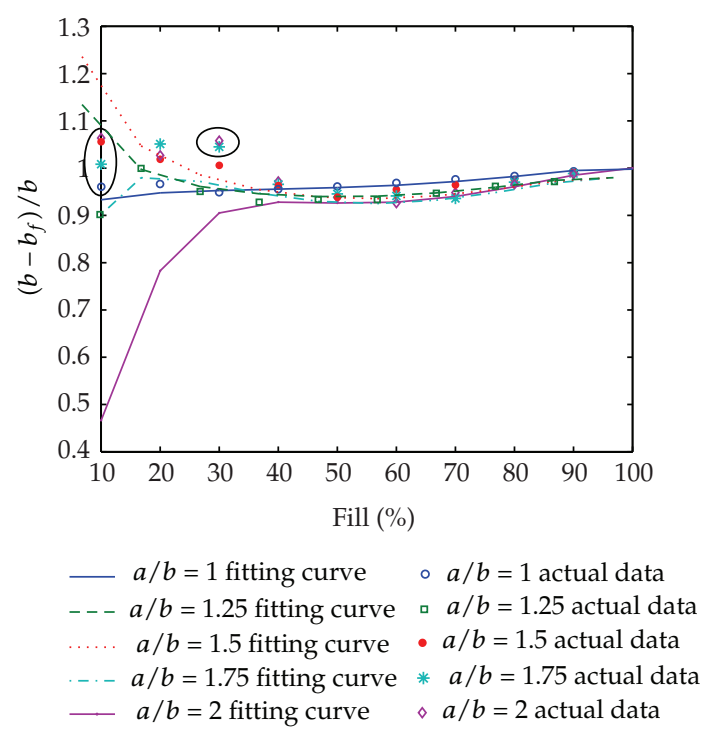

Figure 19: Location of the fixed liquid mass.

For tanks with circular or oval cross-sections, a trammel pendulum mechanical model is derived and parametric expressions for it are obtained through analogy analysis and FLUENT simulations. The establishment of the equivalent mechanical model for lateral liquid sloshing in partially filled tank vehicles has a great importance for accurately analyzing the roll stability of tank vehicles, as well as for developing active/passive roll control systems for them.

The following important discoveries were made from the FLUENT simulations.

(1) For tanks with equal cross-sectional area and liquid fill percentages, tanks with a circular cross-section are subject to the lowest liquid sloshing forces.

(2) For all of the tanks, the maximum liquid sloshing force is produced when the liquid fill percentage is close to $60 \%$. Lower or higher fill percentages cause relatively less sloshing force.

(3) The lower the liquid fill percentage is, the larger the liquid sloshing force produced by per unit of liquid mass is.

Since we make the assumption in deriving the equation of motion for the trammel pendulum that the pendulum amplitude is quite small and the motion of the pendulum is linear, the pendulum model is limited in analyzing liquid sloshing in tank vehicles when the vehicle subjects to gently lateral excitations only. Thus, an equivalent mechanical model for liquid sloshing which can describe nonlinear characteristics will be conducted in a future study.

\section{Acknowledgment}

This paper is supported by Project 20121099 supported by the Graduate Innovation Fund of Jilin University. 


\section{References}

[1] J. Woodrooffe, "Evaluation of dangerous goods vehicle safety performance," Report TP 13678-E, Transport Canada, 2000.

[2] B. P. Douglas, H. Kate, M. Nancy et al., "Cargo tank roll stability study: final report," Tech. Rep. GS23-0011L, U.S. Department of Transportation, 2007.

[3] W. H. Wang, Q. Cao, K. Ikeuchi, and H. Bubb, "Reliability and safety analysis methodology for identification of drivers' erroneous actions," International Journal of Automotive Technology, vol. 11, no. 6, pp. 873-881, 2010.

[4] W. Wang, W. Zhang, H. Guo, H. Bubb, and K. Ikeuchi, "A safety-based approaching behavioural model with various driving characteristics," Transportation Research Part C, vol. 19, no. 6, pp. 12021214, 2011.

[5] R. Ranganathan, S. Rakheja, and S. Sankar, "Steady turning stability of partially filled tank vehicles with arbitrary tank geometry," Journal of Dynamic Systems, Measurement and Control, Transactions of the ASME, vol. 111, no. 3, pp. 481-489, 1989.

[6] X. Kang, S. Rakheja, and I. Stiharu, "Optimal tank geometry to enhance static roll stability of partially filled tank vehicles," SAE Technical Paper 1999-01-3730, 1999.

[7] X. Kang, S. Rakheja, and I. Stiharu, "Cargo load shift and its influence on tank vehicle dynamics under braking and turning," International Journal of Heavy Vehicle Systems, vol. 9, no. 3, pp. 173-203, 2002.

[8] G. Popov, S. Sankar, T. S. Sankar, and G. H. Vatistas, "Dynamics of liquid sloshing in horizontal cylindrical road containers," Proceedings of the Institution of Mechanical Engineers, Part C, vol. 207, no. 6, pp. 399-406, 1993.

[9] H. Akyildiz, "A numerical study of the effects of the vertical baffle on liquid sloshing in two-dimensional rectangular tank," Journal of Sound and Vibration, vol. 331, pp. 41-52, 2012.

[10] S. M. Hasheminejad and M. Aghabeigi, "Liquid sloshing in half-full horizontal elliptical tanks," Journal of Sound and Vibration, vol. 324, no. 1-2, pp. 332-349, 2009.

[11] W. Rumold, "Modeling and simulation of vehicles carrying liquid cargo," Multibody System Dynamics, vol. 5, no. 4, pp. 351-374, 2001.

[12] M. Toumi, M. Bouazara, and M. J. Richard, "Impact of liquid sloshing on the behaviour of vehicles carrying liquid cargo," European Journal of Mechanics, A, vol. 28, no. 5, pp. 1026-1034, 2009.

[13] G. Yan, S. Rakheja, and K. Siddiqui, "Experimental study of liquid slosh dynamics in a partially-filled tank," Journal of Fluids Engineering, Transactions of the ASME, vol. 131, no. 7, Article ID 071303, 14 pages, 2009.

[14] J. A. Romero, O. Ramírez, J. M. Fortanell, M. Martinez, and A. Lozano, “Analysis of lateral sloshing forces within road containers with high fill levels," Proceedings of the Institution of Mechanical Engineers, Part D, vol. 220, no. 3, pp. 302-312, 2006.

[15] K. Modaressi-Tehrani, S. Rakheja, and R. Sedaghati, "Analysis of the overturning moment caused by transient liquid slosh inside a partly filled moving tank," Proceedings of the Institution of Mechanical Engineers, Part D, vol. 220, no. 3, pp. 289-301, 2006.

[16] C. Y. Shang and J. C. Zhao, "Studies on liquid sloshing in rigid containers using FLUENT code," Journal of Shanghai Jiaotong University, vol. 42, no. 6, pp. 953-956, 2008.

[17] F. T. Dodge, "Analytical representation of lateral sloshing by equivalent mechanical models," in The Dynamic Behavior of Liquids in Moving Containers, H. N. Abramson and S. Silverman, Eds., chapter 6, National Aeronautics and Space Administrator, Washington, DC, USA, 1966, NASA SP-106.

[18] H. N. Abramson, W. H. Chu, and D. D. Kana, "Some studies of nonlinear lateral sloshing in rigid containers," Journal of Applied Mechanics, vol. 33, no. 4, 8 pages, 1966.

[19] H. N. Abramson, "The dynamic behavior of liquids in moving containers," NASA SP-106, 1966.

[20] Q. Li, X. Ma, and T. Wang, "Equivalent mechanical model for liquid sloshing during draining," Acta Astronautica, vol. 68, no. 1-2, pp. 91-100, 2011.

[21] M. Utsumi, "A mechanical model for low-gravity sloshing in an axisymmetric tank," Journal of Applied Mechanics, Transactions ASME, vol. 71, no. 5, pp. 724-730, 2004.

[22] J. S. Love and M. J. Tait, "Equivalent linearized mechanical model for tuned liquid dampers of arbitrary tank shape," Journal of Fluids Engineering, vol. 133, Article ID 061105, 7 pages, 2011.

[23] R. Ranganathan, Y. Ying, and J. Miles, "Analysis of fluid slosh in partially filled tanks and their impact on the directional response of tank vehicles," SAE Transactions, vol. 102, pp. 502-505, 1993.

[24] R. Ranganathan, S. Rakheja, and S. Sankar, "Directional response of a B-train vehicle combination carrying liquid cargo," Journal of Dynamic Systems, Measurement and Control, Transactions of the ASME, vol. 115, no. 1, pp. 133-139, 1993. 
[25] M. I. Salem, Rollover stability of partially filled heavy-duty elliptical tankers using trammel pendulums to simulate fluid sloshing [Ph.D. thesis], West Virginia University, Department of Mechanical and Aerospace Engineering, 2000.

[26] L. Dai, L. Xu, and B. Setiawan, "A new non-linear approach to analysing the dynamic behaviour of tank vehicles subjected to liquid sloshing," Proceedings of the Institution of Mechanical Engineers, Part K, vol. 219, no. 1, pp. 75-86, 2005.

[27] http://www.chinacar.com.cn/banguache/peixin_1182/XH9140G_140103.html.

[28] FLUNET 6. 3 User's Guide, FLUENT Inc., 2006. 


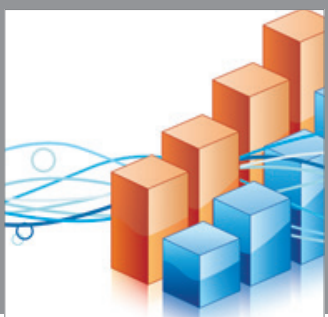

Advances in

Operations Research

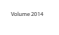

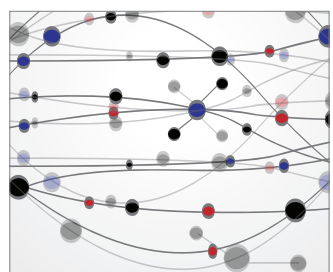

\section{The Scientific} World Journal
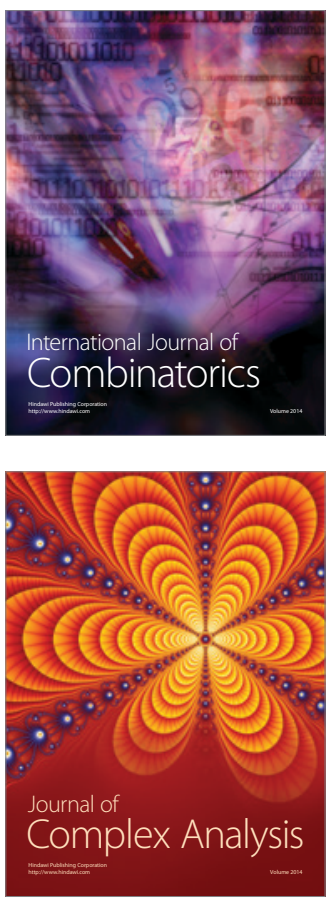

International Journal of

Mathematics and

Mathematical

Sciences
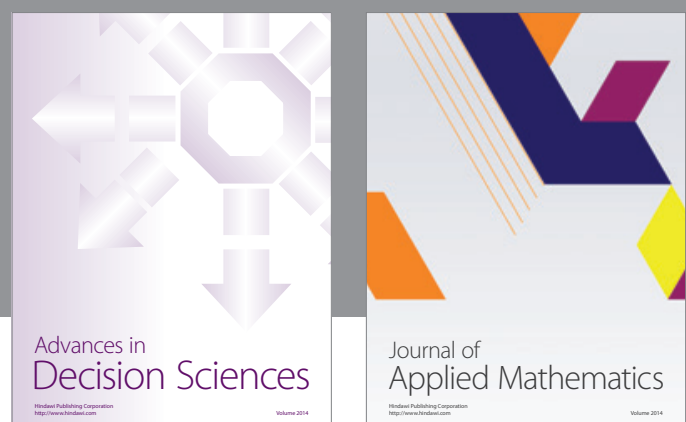

Journal of

Applied Mathematics
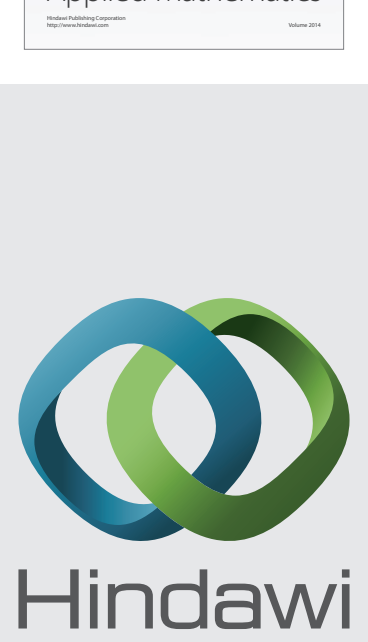

Submit your manuscripts at http://www.hindawi.com
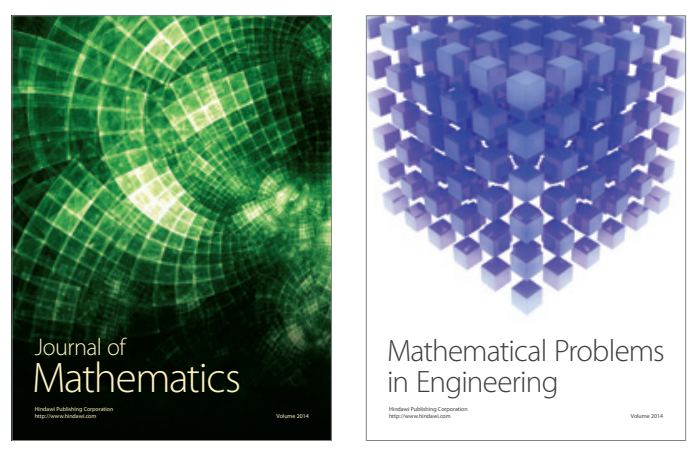

Mathematical Problems in Engineering
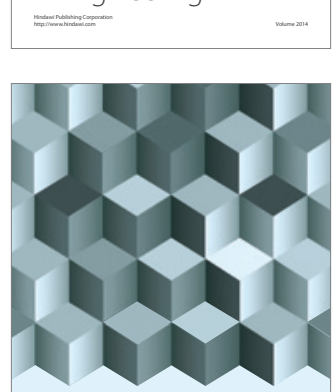

Journal of

Function Spaces
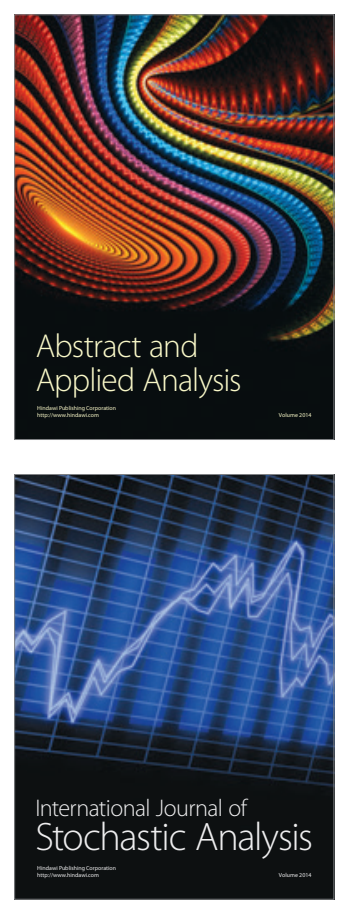

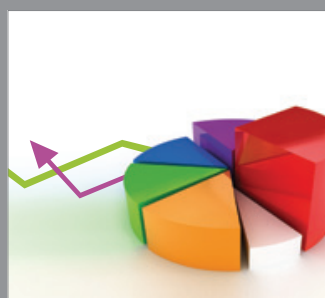

ournal of

Probability and Statistics

Promensencen
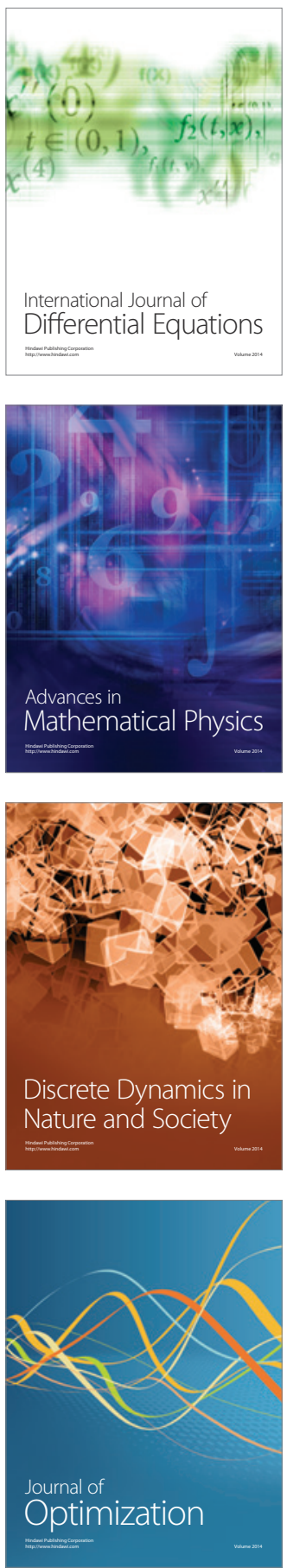